\title{
A Rabbit Hole between Topology and Geometry
}

\author{
David G. Glynn \\ CSEM, Flinders University, P.O. Box 2100, Adelaide, SA 5001, Australia \\ Correspondence should be addressed to David G. Glynn; david.glynn@flinders.edu.au
}

Received 10 July 2013; Accepted 13 August 2013

Academic Editors: A. Ferrandez, J. Keesling, E. Previato, M. Przanowski, and H. J. Van Maldeghem

Copyright (C) 2013 David G. Glynn. This is an open access article distributed under the Creative Commons Attribution License, which permits unrestricted use, distribution, and reproduction in any medium, provided the original work is properly cited.

\begin{abstract}
Topology and geometry should be very closely related mathematical subjects dealing with space. However, they deal with different aspects, the first with properties preserved under deformations, and the second with more linear or rigid aspects, properties invariant under translations, rotations, or projections. The present paper shows a way to go between them in an unexpected way that uses graphs on orientable surfaces, which already have widespread applications. In this way infinitely many geometrical properties are found, starting with the most basic such as the bundle and Pappus theorems. An interesting philosophical consequence is that the most general geometry over noncommutative skewfields such as Hamilton's quaternions corresponds to planar graphs, while graphs on surfaces of higher genus are related to geometry over commutative fields such as the real or complex numbers.
\end{abstract}

\section{Introduction}

The British/Canadian mathematician H.S.M. Coxeter (19072003) was one of most influential geometers of the 20th century. He learnt philosophy of mathematics from $\mathrm{L}$. Wittgenstein at Cambridge, inspired M.C. Escher with his drawings, and influenced the architect R. Buckminster Fuller. See [1]. When one looks at the cover of his book "Introduction to Geometry" [2], there is the depiction of the complete graph $K_{5}$ on five vertices. It might surprise some people that such a discrete object as a graph could be deemed important in geometry. However, Desargues 10-point 10-line theorem in the projective plane is in fact equivalent to the graph $K_{5}$ : in mathematical terms the cycle matroid of $K_{5}$ is the Desargues configuration in three-dimensional space, and a projection from a general point gives the configurational theorem in the plane. Desargues theorem has long been recognised (by Hilbert, Coxeter, Russell, and so on) as one of the foundational theorems in projective geometry. However, there is an unexplained gap left in their philosophies: why does the graph give a theorem in space? Certainly, the matroids of almost all graphs are not theorems. The only other example known to the author of a geometrical theorem coming directly from a graphic matroid is the complete bipartite graph $K_{3,3}$, which gives the 9-point 9-plane theorem in three-dimensional space; see [3]. It is interesting that both
$K_{5}$ and $K_{3,3}$ are minimal nonplanar (toroidal) graphs and both lead to configurational theorems in the same manner.

In this paper, we explain how virtually all basic linear properties of projective space can be derived from graphs and topology. We show that any map (induced by a graph of vertices and edges) on an orientable surface of genus $g$, having $v$ vertices, $e$ edges, and $f$ faces, where $v-e+f=$ $2-2 g$, is equivalent to a linear property of projective space of dimension $v-1$, coordinatized by a general commutative field. This property is characterized by a configuration having $v+f$ points and $e$ hyperplanes. This leads to the philosophical deduction that topology and geometry are closely related, via graph theory. If $g=0$ (and the graph is planar), the linear property is also valid for the most general projective spaces, which are over skewfields that in general have noncommutative multiplication. This is a powerful connection between the topology of orientable surfaces and discrete configurational properties of the most general projective spaces.

There are various "fundamental" theorems that provide pathways between different areas of mathematics. For example, the fundamental theorem of projective geometry (FTPG) describes the group of automorphisms of projective geometries over fields or skewfields (all those of dimensions greater than two) as a group of nonsingular semilinear transformations. This most importantly allows the choice of coordinate systems in well-defined ways. Hence, the FTPG is 
a pathway between projective geometry and algebra, matrix, and group theory.

Another example is the fundamental theorem of algebra. This provides another pathway between polynomials of degree $n$ over the real number field and multisets of $n$ roots, which are complex numbers. It explains why the complex numbers are important for an understanding of the real numbers.

In a similar vein we show here how our "rabbit hole" between topology and geometry can be used to obtain the basic properties of the most general projective geometry directly from topological considerations.

Here is an outline of the approach.

(1) Consider the properties of fundamental configurations in $(v-1)$-dimensional projective geometry, which are collections of points and hyperplanes with incidences between them. The most important have $v$ points on each hyperplane, and these points form a minimal dependent set (a "circuit" in matroid theory).

(2) In most of these configurations, the algebraic property that corresponds to a configurational theorem is that a set of $e$ subdeterminants of size two in a general $v \times f$ matrix over a field has a linear dependency; that is, the vanishing of any $e-1$ subdeterminants implies the vanishing of the remaining subdeterminant.

(3) The condition for such a set of subdeterminants is topological: the dependency amongst the subdeterminants happens if and only if there exists a graph having $v$ vertices and $e$ edges embedded on an orientable surface of genus $g$ and inducing $f$ faces (certain circuits of the graph that can be contracted to a point on the surface).

(4) A bonus is that when the surface has genus zero (i.e., the graph is planar), the commutative field restriction for the algebraic coordinates of the space can be relaxed to noncommutative skewfields including the quaternions. This requires a different interpretation for a $2 \times 2$ determinant and another proof depending upon topological methods.

(5) Since the latter method of planar graphs produces the main axiom for projective geometry (the bundle theorem or its dual Pasch axiom; see [4, page 24]) and the former one for standard determinants over commutative fields produces the Pappus theorem, we see that all bases are covered, and a topological explanation for standard projective geometry, that is, embeddable into space of dimension greater than two, is obtained. In the case of 2-dimensional geometries (planes) there exist non-Desarguesian projective planes so these geometries do not appear to be produced topologically; see [5, page 120] and [6, Section 23].

\section{Definitions and Concepts}

Let us summarize the topological and geometrical concepts that are used in this paper. A graph is a collection of vertices with a certain specified multiset of edges, each of which is a multiset containing two vertices. If a vertex is repeated, then the edge is a loop. The graph is simple if it contains no loop and no multiple edges, edges that are repeated.

An orientable surface is a surface in real threedimensional space that can be constructed from the sphere by appending $g$ handles; see [2, Section 21.1]. This surface has $g$ holes, and we say that it has genus $g$. One classical use for such a surface is to parametrize the points on an algebraic curve in the complex plane, but we have another application in mind.

A skewfield or division ring is an algebraic structure $(F,+, \cdot)$, where $F$ is a set containing distinct elements 0 and 1 , for which $(F,+)$ is an abelian (i.e., commutative) group, with identity 0 , and $\left(F^{*}:=F \backslash\{0\}, \cdot\right)$ is a group (nonabelian if the skewfield is "proper"). The left and right distributive laws $a(b+c)=a b+a c$ and $(a+b) c=a c+b c$ hold, for $\forall a, b, c \in F$. The classical example of a proper skewfield is the quaternion system of Hamilton (four-dimensional over the reals). If the multiplicative group $F^{*}$ is abelian (i.e., commutative), $F$ is called a field. Thus a field is a special case of skewfield. Classical examples of a field are the rational numbers, the real numbers, and the complex numbers. It is known (by Wedderburn's theorem and elementary field theory) that the only finite skewfields are the Galois fields $\mathrm{GF}(q)$, where $q$ is a power of a prime.

A projective geometry of dimension $n$ over a skewfield is the set of subspaces of a (left or right) vector space of rank $n+1$ over the skewfield. Points are subspaces of projective dimension zero, while hyperplanes are subspaces of projective dimension $n-1$. It is well-known (or by the FTPG) that every projective space of dimension at least three has a coordinatization involving a skewfield and comes from the relevant vector space. There are some incidence properties for geometries over fields that are not valid for those over the more general skewfields. For example, the bundle theorem is valid for skewfields (and fields), but Pappus $9_{3}$ theorem only holds for geometries over fields.

It is known that certain of the configurational theorems are in some sense "equivalent" in that assuming any one of them implies the remaining ones. These include the theorems of Pappus, Möbius, and Gallucci. These latter theorems are all explained by the present topological theory. Desargues theorem and the bundle theorem (or its dual, the configuration of Pasch) are also in some sense equivalent in the case of the more general geometries over skewfields; see [6]. We show that the bundle theorem comes from the topology of planar graphs.

An abstract configuration is a set of points and a distinguished collection of subsets, called blocks. An embedding of such a configuration is a way of putting the points into a projective space so that each of the blocks generates a hyperplane and not the whole space. The point-set as a whole should generate the whole projective space. There are several ways of thinking about embeddings (e.g., often they may have more incidences than specified by the abstract configuration), and we refer the reader to [7] for a discussion. However, extra incidences do not bother us here.

Our configurations have blocks with all the same size $k$. We say that such a configuration is a configurational theorem if for each embedding of the configuration into space of 


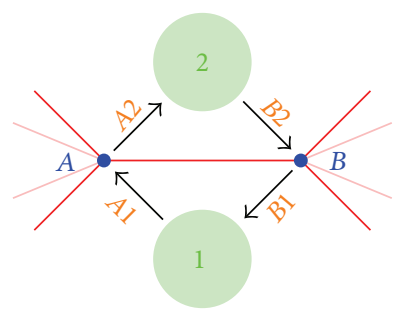

Figure 1: Graph fragment.

dimension $k-1$, the property that all but one of the blocks lie in hyperplanes implies the same is true for the remaining block. This might hold only for spaces over fields but not general skewfields, as with Pappus theorem.

\section{Main Results}

We present two main results. Theorem 1 relates graphs or maps on orientable surfaces of any genus to configurational theorems in general projective space over any commutative field (such as the rational numbers, real numbers, complex numbers, or finite fields). This uses $2 \times 2$ determinants with the standard definition. However, for general skewfields this definition of determinant does not work, and so we use Lemma 2 to find an alternate way and find that there is a restriction to surfaces of genus zero. Thus, Theorem 4 investigates the graphs or maps on a surface of genus zero and relates them to configurational theorems over skewfields.

Theorem 1. Any graph $G$ embedded on an orientable surface of genus $g \geq 0$, having $v$ vertices, e edges, and $f$ faces, where by Euler's formula $v-e+f=2-2 g$, is equivalent to a certain configurational theorem (explained in the proof) in projective space $P G(v-1, F)$, where $F$ is any commutative field.

Proof. Let us label the vertices of $G$ with the letters $A, B, C, \ldots$ in a set $V$ of cardinality $v$ and label the faces (which are certain circuits on the surface) with the natural numbers $1,2,3, \ldots, f$. Then, each of the $e$ edges of the graph joins precisely two vertices, for example, $A$ and $B$, and it forms part of the boundaries of precisely two faces, for example, 1 and 2. (For simplicity we are assuming that there are no loops in both the graph and its dual, but these can easily be accounted for in a more expansive theory.) Note that the dual graph $G^{d}$ is the graph embeddable on the same surface where we switch the roles of vertex and face, joining two faces if they have a common edge. This dual graph depends strongly upon the embedding, so that a graph may have different dual graphs on other surfaces: see [8] for recent research on this topic.

We define an abstract configuration $K$ having $v+f$ points and $e$ blocks, which are subsets of $v$ points as follows. The points are identified with $V \cup\{1, \ldots, f\}$, that is, the union of the set of points and the set of faces of $G$. Additionally, for each edge $A B$ bounded by the two faces 1 and 2, there is the corresponding set of $v$ points which is $\overline{A B} 12:=V \backslash\{A, B\} \cup$ $\{1,2\}$; that is, we replace $A$ and $B$ in $V$ by 1 and 2 , and we call this a block of $K$.
Consider any $v \times f$ matrix $M$ over a field $F$ (where the multiplication is commutative, i.e. $x y=y x$ for all $x, y \in F)$, with rows in correspondence with the vertices of $G$ $(A, B, \ldots)$ and the columns in correspondence with the faces of $G(1,2, \ldots, f)$. We assume that a typical matrix element corresponding to vertex $C$ and face $i$ has $m_{C i}=m_{i C}$. Thus, the subscripts are treated like unordered sets $\{C, i\}$. For any "graph fragment" corresponding to an edge $A B$ of $G$, see Figure 1, there is a $2 \times 2$ submatrix of $M$ in the rows $A$ and $B$ and in the columns 1 and 2. The "angles" $A 1, B 1, A 2$, and $B 2$ correspond to the four positions in the submatrix, while the determinant of this submatrix is $m_{A 1} \cdot m_{B 2}-m_{A 2} \cdot m_{B 1}$. In a general embedding of $K$ into $\operatorname{PG}(v-1, F)$, we may assume that the points from $V$ form a basis and so are coordinatized by the unit vectors. If the remaining points of $K$ had no constraints upon them except for being embedded in $\operatorname{PG}(v-1, F)$, they would be coordinatized by completely general (nonzero) vectors of length $v$ and realized by the $f$ columns of the matrix $M$. Then the vanishing of the subdeterminant corresponding to the edge $A B$ is found to be equivalent to the fact that the $v$ points $\overline{A B} 12$, as defined above, lie in a hyperplane.

Since the surface of $G$ is orientable, we may orient it so that at each vertex there is an anticlockwise direction. The equivalence between cyclic graphs, graphs in which there is a cyclic order at each vertex, and embeddings of graphs on such surfaces has been discussed by many people, starting apparently with Heffter [9] and later clarified by Edmonds [10]. They have been given many names, such as graphs with rotation systems, ribbon graphs, combinatorial premaps, and fatgraphs: see [11-13]. Consider Figure 1 again. Small anticlockwise-oriented circles around $A$ and $B$ induce a larger clockwise-oriented circle going from $A \rightarrow 2 \rightarrow B \rightarrow 1 \rightarrow$ $A$. Thus, given any edge of $G$ containing a vertex $C$ and being the boundary of a face $i$, this orients the angle from vertex $C$ to face $i$ or from $i$ to $C$. Denote these possibilities by $C i$ or $i C$, respectively. However, such an angle occurs with precisely two edges, and one edge gives $C i$ and the other $i C$.

The $2 \times 2$ subdeterminant, with rows $A$ and $B$ and columns 1 and 2 , may be written $m_{1 A} \cdot m_{2 B}-m_{A 2} \cdot m_{B 1}$, according to the clockwise orientation. (We purposely forget for a while that $m_{i C}=m_{C i}$.) Now the vanishing of this determinant is equivalent to $m_{1 A} \cdot m_{2 B}=m_{A 2} \cdot m_{B 1}$ (we could call the two sides of this equation the "diagonals" of the determinant), and if all the determinants corresponding to the edges of $G$ vanish, we can take the product over all $e$ edges on both sides to obtain $\Pi_{A B \in G} m_{1 A} \cdot m_{2 B}=\Pi_{A B \in G} m_{A 2} \cdot m_{B 1}:=p$. This is clearly a trivial identity since any angle, for example, $i C$, occurs once on the left and once (as $\mathrm{Ci}$ ) on the right. Now we can assume that the "angle variables" $m_{i C}$ are all nonzero, as otherwise there will be an unwanted hyperplane in $K$ which would not be in the most general position. Then the vanishing of any $e-1$ of the subdeterminants implies the vanishing of the remaining one, since we can divide $p$ by $e-1$ "diagonals" $m_{1 A} \cdot m_{2 B}$ on the left and by the corresponding $e-1$ "diagonals" $m_{A 2} \cdot m_{B 1}$ on the right, and we obtain the vanishing of the last determinant. This shows the theorem in the general case where $F$ is a field with commutative multiplication. 
The converse construction holds: a configurational theorem in space that relies on $2 \times 2$ matrices as above must come from a graph on an orientable surface. The problem is to determine the cyclic graph $G$ from the set of $e 2 \times 2$ subdeterminants of a matrix having the property that the vanishing of any $e-1$ of them implies that the remaining subdeterminant vanishes. Around the edges of each vertex of $G$ there should be an anticlockwise cyclic orientation or "cyclic order." If we start with a vertex $A$ and an edge $\{A, B\}$ containing it, proceed to the next edge $\{A, C\}$ in the cyclic order, and using the cyclic order at $C$, find the next edge $\{C, D\}$, and so on, we should follow around all the edges of a face of the embedding in a clockwise way and return to the first vertex $A$ and edge $\{A, B\}$. We will show how this is achieved. Now, as before, we can assume that the entries, where the subdeterminants occur, are all nonzero. If the subdeterminants have the assumed property, they can be ordered so that one "diagonal" of each is selected, and the product of all these selected diagonals is the same as the product of the nonselected ones (as in the first part of the proof above). As before we may write the selected diagonals in the form $m_{1 A} \cdot m_{2 B}$ and the nonselected ones in the form $m_{A 2} \cdot m_{B 1}$. To find the graph we must associate the rows of the matrix $M$ with the vertices, the columns with faces, and the subdeterminants with the edges. Consider a particular vertex $A$ of $G$ (a row of $M$ ). We obtain a cyclic (anticlockwise) chain of $n$ subdeterminants using that row (equivalently, edges of $G$ containing $A$ ) as follows: $d:=m_{1 A}$. $m_{2 B}-m_{A 2} \cdot m_{B 1}, e:=m_{2 A} \cdot m_{3 C}-m_{A 3} \cdot m_{C 2}, f:=m_{3 A} \cdot m_{4 D}-m_{A 4}$. $m_{D 3}, \ldots, g:=m_{n A} \cdot m_{1 E}-m_{A 1} \cdot m_{E n}$. Now we can check that the faces of $G$ also arise from this construction. Starting with the vertex $A$ and edge containing it $d=m_{1 A} \cdot m_{2 B}-m_{A 2} \cdot m_{B 1}$, the next edge determinant in $A$ 's anticlockwise order from $d$ is $e:=m_{2 A} \cdot m_{3 C}-m_{A 3} \cdot m_{C 2}$ which contains the vertexrow $C$. The cyclic ordering at $C$ makes $m_{2 C} \cdot m_{k F}-m_{C k} \cdot m_{F 2}$ the next edge (for some vertex-row $F$ and column-face $k$ ). Following this sequence of subdeterminants (edges) around we see that the edges surround the column-face 2, and we can say that the cyclic ordering induced on the edges of the face in this way is clockwise. So it works out similarly given any vertex and edge containing that vertex. However, one might see a minor problem with this argument. In a standard (cyclic) graph $G$ there should be one cycle (of edges) at each vertex: if there are $x_{r}$ cycles determined by a row $A$ of $M$, we "split" that row into $x_{r}$ distinct rows, one for each disjoint cycle of subdeterminants with $A$. Similarly we look at each column $c$, and there will be $y_{c}$ disjoint cycles on the rows induced by the subdeterminants with that column. Splitting that column into $y_{c}$ distinct columns will enable us to look at a larger matrix with the same number of subdeterminants, but with each row and column corresponding to a unique cycle. Subdeterminants in different cycles will not have rows or columns in common. Then the graph on the orientable surface has $\Sigma_{r} x_{r}$ vertices and $\Sigma_{c} y_{c}$ faces. The other way around, given a set of $2 \times 2$ determinants with our special property, if we collapse the matrix by identifying certain rows or columns, then the property is retained, as long as we do not identify two rows or columns belonging to the same subdeterminant. By this process cycles of subdeterminants can be created with the same row or column. Geometrically, it is the same as creating a new geometrical theorem by identifying points or hyperplanes. However, these examples can then be expanded out again by splitting the rows or columns into bigger collections of rows or columns as above, and the pattern of subdeterminants in the largest matrix is canonical up to permutations of rows and columns. So we see how to get around this minor problem in the proof.

What kind of configurational theorems $K$ corresponds to graphs on orientable surfaces? One obvious condition is that the configuration must have $v+f$ points in $\mathrm{PG}(v-1, F)$. There are $e$ hyperplanes or blocks in $K$, each containing $v$ points. More importantly, there should be a subset $V$ of $v$ points in $K$ such that each hyperplane of $K$ contains precisely $v-2$ points of $V$ and two others.

Now we explain the noncommutative case which is related to planar graphs.

Lemma 2. Let $F$ be a skewfield with perhaps noncommutative multiplication. The condition that a set of $v$ points of $P G(v-$ $1, F)$, consisting of $A, B$, and the unit vectors $e_{3}, \ldots, e_{v}$, is contained in a hyperplane is a "cyclic identity" $a^{-1} b c^{-1} d=1$, where $\left(\begin{array}{ll}a & b \\ d & c\end{array}\right)$ is a certain $2 \times 2$ matrix over $F$. (Here we are assuming a "generic" case where all the $a, b, c, d$ are nonzero.)

Proof. A point of $\operatorname{PG}(v-1, F)$ is a nonzero column vector with $v$ coefficients from $F$ that are not all zero. Two of these column vectors $\mathbf{y}$ and $\mathbf{z}$ give the same point if one can find a nonzero element $f \in F$ such that $\mathbf{y}=\mathbf{z} f$. The hyperplanes of $\mathrm{PG}(v-1, F)$ can be coordinatized by row vectors of length $v$ over $F$, in a similar way to the points. Then a point $\mathbf{y}$ is contained in a hyperplane $\mathbf{h}$ if and only if $\mathbf{h y}=0$, (h is a row and $\mathbf{y}$ is a column vector). Notice that here we are multiplying points on the left (and hyperplanes on the right). Thus we must restrict ourselves to operations on the points of $\mathrm{PG}(v-$ $1, F)$ that act on the left. A square $v \times v$ matrix is "singular" (and its column points are in a hyperplane) if and only if it cannot be row-reduced (by multiplying on the left by a square matrix) to the identity matrix, or equivalently, it can be row reduced so that a zero row appears. In our situation we have a $v \times v$ matrix that consists of $v-2$ different unit vectors and a $2 \times 2$ two submatrix $X=\left(\begin{array}{ll}a & b \\ d & c\end{array}\right)$ (with $a, b, c, d$ all nonzero) in the remaining part row disjoint from the ones of the unit vectors. We can then restrict our row reductions to the two rows of $X$, and we see that the whole matrix is singular if and only if $X$ is singular. It is still not possible to use the ordinary determinant to work out if $X$ is singular. But assuming that both $a$ and $d$ are nonzero we may multiply the first row by $a^{-1}$ and the second by $d^{-1}$. This leaves us with the matrix

$$
\left(\begin{array}{ll}
1 & a^{-1} b \\
1 & d^{-1} c
\end{array}\right),
$$

and the condition for singularity of this matrix is clearly $a^{-1} b=d^{-1} c$, as then we can further row-reduce to obtain a zero row. This gives the "cyclic condition" $a^{-1} b c^{-1} d=1$ (= $\left.d a^{-1} b c^{-1}=c^{-1} d a^{-1} b=b c^{-1} d a^{-1}\right)$, if $c$ is also nonzero.

Note that $a^{-1} b=d^{-1} c$ does not imply that $a b^{-1} c d^{-1}=$ 1: equivalently, transposing a general $2 \times 2$ matrix over 
a skewfield does not always preserve its singularity. There is quite a lot of theory about determinants for skewfields, see for example, $[14,15]$, but we can have a more elementary approach here since we only deal with $2 \times 2$ subdeterminants.

This leads us to consider a special type of planar graph that has cyclic identities at each vertex. It is well known that any planar graph with an even number of edges on each face is bipartite; see, for example, [8]. By dualizing this statement we also know that any planar graph which is Eulerian, that is, has an even valency at each vertex, has a bipartite dual. What this means is that the edges of such a planar Eulerian graph may be oriented so that the edges on each face go in a clockwise or in an anticlockwise direction. Then, if we travel around any vertex in a clockwise direction, the edges alternate, going out and into the vertex. We call such an orientation Eulerian.

In general, an Eulerian orientation of a graph having even valency at each vertex is an orientation of each edge (put an arrow on the edge) such that there are equal numbers of edges going out or into each vertex. For the above embedding in the plane we find a natural Eulerian orientation that is determined by the faces.

Lemma 3. Consider a planar graph $H$ with a bipartite dual having its Eulerian orientation of the edges. Then there is noncommutative cyclic identity with variables over any skewfield at each vertex, and any one of these cyclic identities is implied by the remaining cyclic identities.

Proof. Consider the list of edges $E$, and for each $e \in E$ let $e=$ $(A, B)$, where the Eulerian orientation goes from vertex $A$ on $e$ to vertex $B$ on $e$. The "cyclic identity" at vertex $A$ is of the form $x_{e_{1}}^{-1} \cdot x_{e_{2}} \cdots x_{e_{2 d-1}}^{-1} \cdot x_{e_{2 d}}=1$, where the edges of the graph on $A$ are (in the clockwise ordering around $A) e_{1}, e_{2}, \ldots, e_{2 d}$, where $e_{1}=(A, B), e_{2}=(C, A), e_{3}=(A, D), \ldots, e_{2 d}=(X, A)$. Note that if we had have started with any other edge, for example, $e_{3}$, going out from $A$, we would have obtained an equivalent identity, since by multiplying both sides on the left by $x_{e_{2}}^{-1} x_{e_{1}}$ and then both sides on the right by $x_{e_{1}}^{-1} x_{e_{2}}$ we obtain

$$
\begin{gathered}
x_{e_{1}}^{-1} \cdot x_{e_{2}} \cdots x_{e_{2 d-1}}^{-1} \cdot x_{e_{2 d}}=1 \\
\Longrightarrow x_{e_{3}}^{-1} \cdot x_{e_{4}} \cdots x_{e_{2 d-1}}^{-1} \cdot x_{e_{2 d}}=x_{e_{2}}^{-1} x_{e_{1}} \\
\Longrightarrow x_{e_{3}}^{-1} \cdot x_{e_{4}} \cdots x_{e_{2 d-1}}^{-1} \cdot x_{e_{2 d}} \cdot x_{e_{1}}^{-1} \cdot x_{e_{2}}=1 .
\end{gathered}
$$

Now consider any face of the graph with its clockwise or anticlockwise orientation. If it has $n$ vertices (in the cyclic order labelled $A_{1}, \ldots, A_{n}$ ), then there are $n$ cyclic identities attached. Consider the operation of collapsing the face down to a single vertex and erasing all the edges of the face. The cyclic identities can be multiplied in the cyclic order so that a new cyclic identity is obtained. If a loop having adjacent ins and outs at a vertex appears, then it may be safely purged from the graph, since there can be no holes in the surface and since in the cyclic identity at the vertex the edge variable will cancel with itself. The new collapsed graph has cyclic identities that derive from the larger graph. By continuing this process we obtain eventually a planar graph with two vertices $A$ and $B$ joined by an even number $2 d$ of edges. If the cyclic identity at

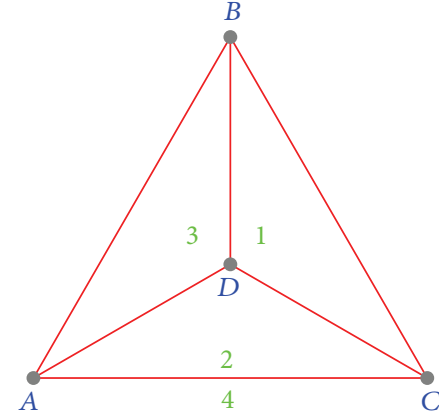

Figure 2: The tetrahedron (graph of the bundle theorem) in the plane.

$A$ is $x_{1}^{-1} \cdot x_{2} \cdots x_{2 d-1}^{-1} \cdot x_{2 d}=1$, with the odd edges directed from $A$ to $B$ and the even edges from $B$ to $A$, then the clockwise order at $B$ will be the reverse of that at $A$, and so the cyclic identity at $B$ will be $x_{2 d}^{-1} \cdot x_{2 d-1} \cdots x_{2}^{-1} \cdot x_{1}=1$ which is the inverse identity to that at $A$ and so equivalent to it. Hence the dependency among all the cyclic identities of the original graph is established.

Theorem 4. Any graph $G$ embedded on an orientable surface of genus $g=0$, having $v$ vertices, $e$ edges, and $f$ faces, where by Euler's formula $v-e+f=2$, is equivalent to a configurational theorem in projective space $P G(v-1, F)$, where $F$ is any skewfield or field.

Proof. First we construct the configuration $K$ from the graph $G$ in precisely the same manner as Theorem 1 .

When the graph $G$ is embedded in any orientable surface, which in the present case is now the plane (or the sphere), there is a natural cyclic structure at each vertex. We now go to a graph that is intermediate between $G$ and its dual $G^{d}$. This is called the "medial" graph $M(G)$, and it has $v^{\prime}=e$ vertices and $f^{\prime}=v+f$ faces. It is 4-regular, in that every vertex is joined to four others. Since each edge has two vertices, it is easy to see that the medial graph has $e^{\prime}=2 v^{\prime}$ edges. Notice that since $v-e+f=2-2 g$ (Euler's formula) we have in the medial graph with $v^{\prime}-e^{\prime}+f^{\prime}=v^{\prime}-2 v^{\prime}+f^{\prime}=f^{\prime}-v^{\prime}=v+f-e=2-2 g$ : it is clear the medial graph is also embedded on the same surface as $G$.

For example, if $G$ is the planar tetrahedral graph of Figure 2, then $M(G)$ is the planar octahedral graph, having six vertices and eight faces.

In detail, the set of vertices of $M(G)$ is $\left\{v_{A B}\right.$ । $A B$ edge of $G$, and $v_{A B}$ is joined with $v_{B C}$ in $M(G)$ when $A B$ and $B C$ are adjacent to the same face $f$ of $G$ on the surface: they are also adjacent in the cyclic order at $B$ and in that of $f$. The dual of this medial graph is always bipartite so that there are two types of faces, corresponding to the vertices and to the faces of the original graph $G$. (Conversely, a 4-regular graph on an orientable surface, for which the dual graph is bipartite, is easily seen to be the medial graph of a unique graph on that surface.)

Consider Figure 1, and adjoin $C$ and $D$, which are the vertices in $G$ adjacent to $A$ on the boundaries of faces 1 and 2 , respectively, and adjoin $E$ and $F$ which are the vertices 
TABLE 1: A table of five geometrical theorems.

\begin{tabular}{|c|c|c|c|c|c|c|}
\hline Name & Graph: $v, e, f$ & Dual & Surface: $g$ & P's & $H^{\prime} \mathrm{s}$ & Space \\
\hline Bundle Thm & $K_{4}: 4,6,4$ & $K_{4}$ & Plane: 0 & 8 & 6 & $\mathrm{PG}(3, H)$ \\
\hline Pappus $9_{3} \mathrm{Thm}$ & $3 K_{3}: 3,9,6$ & $K_{3,3}$ & Torus: 1 & 9 & 9 & PG $(2, F)$ \\
\hline Möbius $8_{4}$ Thm & $2 C_{4}: 4,8,4$ & $2 C_{4}$ & Torus: 1 & 8 & 8 & $\mathrm{PG}(3, F)$ \\
\hline Other $8_{4} \mathrm{Thm}$ & $K_{4}+2 e: 4,8,4$ & $K_{4}+2 e$ & Torus: 1 & 8 & 8 & $\operatorname{PG}(3, F)$ \\
\hline Gallucci's Thm & $2 K_{4}: 4,12,8$ & Cube & Torus: 1 & 12 & 12 & PG $(3, F)$ \\
\hline
\end{tabular}

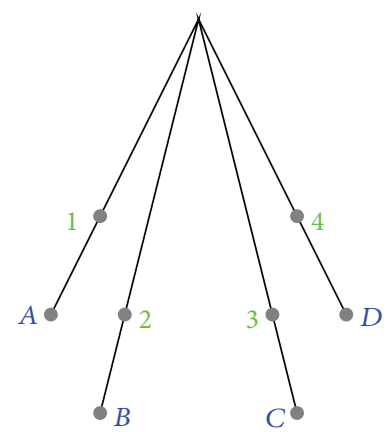

(a)

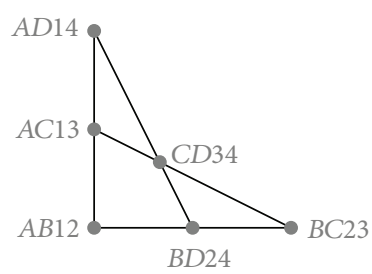

(b)
Figure 3: The bundle theorem in 3d space and its dual Pasch axiom.

adjacent to $B$ on the boundaries of faces 2 and 1 . We see that $v_{A B}$ is joined in the medial graph $M(G)$ with the four vertices $v_{A C}, v_{A D}, v_{B E}$, and $v_{B F}$ in the clockwise direction. Notice that these edges of $M(G)$ are in bijective correspondence with the "angles" $A 1, A 2, B 2, B 1$, respectively. Also, as in the proof of Theorem 1 the selection of "diagonals" of the determinants $m_{1 A} \cdot m_{2 B}-m_{A 2} \cdot m_{B 1}$ at each edge implies that we can orientate the edge $\left(v_{A B}, v_{A C}\right)$ in $M(G)$ and label it with $m_{1 A}^{-1}$; similarly the directed edge $\left(v_{A B}, v_{A E}\right)$ is labelled $m_{2 B}^{-1}$. Then the remaining unselected diagonal of the determinant gives two edges of $M(G)$ directed the other way: $\left(v_{A D}, v_{A B}\right)$ is labelled $m_{A 2}$ and $\left(v_{B F}, v_{A B}\right)$ is labelled $m_{B 1}$. Repeating this for all edges of $G$ we obtain an Eulerian orientation, and each vertex of $M(G)$ corresponds to a cyclic identity with four variables which is equivalent to the determinant condition. For the edge $A B$ above the "cyclic" identity is $m_{1 A}^{-1} \cdot m_{A 2} \cdot m_{2 B}^{-1} \cdot m_{B 1}=1$.

Applying Lemma 3 to the medial graph $M(G)$ we see that the final cyclic identity is dependent upon the others, and so we have proved that $K$ is a configurational theorem for every skewfield and therefore also for every field.

\section{Examples of Configurational Theorems}

If a graph on an orientable surface $S$ gives a configurational theorem $K$, then the dual graph on $S$ gives a configurational theorem that is the matroid dual of $K$. It corresponds to the simple process of transposing the $v \times f$ matrix $M$ containing the subdeterminants in the construction.

Table 1 summarizes the five examples of this section.

4.1. The Bundle Theorem. The bundle theorem in threedimensional projective space is a theorem of eight points and six planes. See Figure 3.
The bundle theorem states that if four lines are such that five of the unordered pairs of the lines are coplanar, then so is the final unordered pair. Translating this to a theorem about points and planes, we can define a line as the span of a pair of distinct points. Thus the lines correspond to pairs of points, and the theorem is about eight points and six planes. It turns out that the configuration is in three-dimensional space, and the four lines must be concurrent.

The dual in terms of points and lines is that if four lines in space have five intersections in points, then so is the sixth intersection. Then all the lines are coplanar. This is the "Axiom of Pasch"; see for example, [4], and it is one of the fundamental axioms from which all the other basic properties derive.

Comparing Figure 2 with Figure 3 the bundle theorem is seen to be the configurational theorem that arises from the tetrahedral graph or equivalently the complete graph $K_{4}$, embedded in the plane.

Relating this to the proof of Theorem 4, the medial graph of $K_{4}$ is the octahedral graph having six vertices and eight faces. Thus the theorem shows that the bundle theorem is valid for all projective geometries of dimension at least three. This leads to the philosophic conclusion that projective geometry and our perceptions of linear geometry may have topological origins.

It is noted that the dual graph of the octahedral graph (in the plane) is the cube, which has eight square faces and six vertices.

The six blocks of four points obtained from the edges of the graph are

$$
\begin{aligned}
& \overline{A B} 34=C D 34, \\
& \overline{A C} 24=B D 24, \\
& \overline{A D} 23=B C 23, \\
& \overline{B C} 14=A D 14, \\
& \overline{B D} 13=A C 13, \\
& \overline{C D} 12=A B 12 .
\end{aligned}
$$

The eight points of this "bundle" theorem in $3 \mathrm{~d}$ space are members of the set $\{A, B, C, D, 1,2,3,4\}$, while the six blocks (contained in planes) are in correspondence with the six edges of the $K_{4}$ graph (the tetrahedron); see Figure 2 .

In the Pasch configuration on the right of Figure 3 , there are again four lines which we could label $A 1, B 2, C 3, D 4$. Each pair of lines intersect in a point, for example, $A 1$ and $B 2$ intersect in the point labelled $A 1 B 2$. The intersection of the 


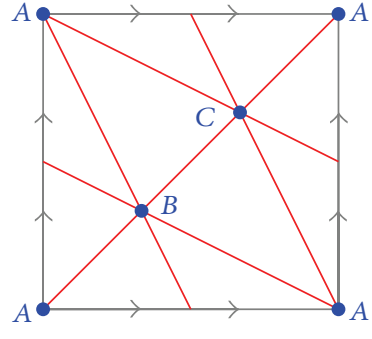

(a)

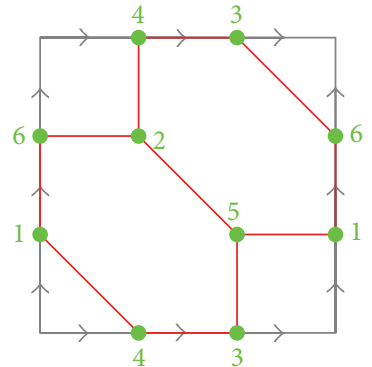

(b)
FIgure 4: The toroidal Pappus graph $3 C_{3}$ and its dual $K_{3,3}$.

final pair of lines $B 2$ and $C 3$ is a consequence of the other intersections. So we verify that the geometric dual of the bundle theorem is the Pasch configuration.

4.2. The Pappus Theorem. The nine points of the Pappus $9_{3}$ configurational theorem in the plane are members of the set $\{A, B, C, 1,2,3,4,5,6\}$, while the nine blocks (contained in lines when the configuration is embedded in the plane) are in correspondence with the nine edges of the $3 C_{3}$ graph; see Figure 4.

The nine blocks obtained from the edges of the graph are

$$
\begin{aligned}
& \overline{A B} 14=C 14, \\
& \overline{A B} 26=C 26, \\
& \overline{A B} 35=C 35, \\
& \overline{B C} 16=A 16, \\
& \overline{B C} 25=A 25, \\
& \overline{B C} 34=A 34, \\
& \overline{C A} 15=B 15, \\
& \overline{C A} 24=B 24, \\
& \overline{C A} 36=B 36 .
\end{aligned}
$$

There are many references for this configuration which dates back to Pappus of Alexandria circa $330 \mathrm{CE}$; see [2, 3, $5,16-18]$. Perhaps the easiest way to construct it in the plane is first to draw any two lines. Put three points on each and connect them up with six lines in the required manner; see Figure 5.

4.3. The Möbius Theorem. The eight points of the Möbius $8_{4}$ configurational theorem in $3 \mathrm{~d}$ space are members of the set $\{A, B, C, D, 1,2,3,4\}$, while the eight blocks (contained in planes when the configuration is in $3 \mathrm{~d}$ space) are in correspondence with the eight edges of the $2 C_{4}$ graph; see Figure 6 .

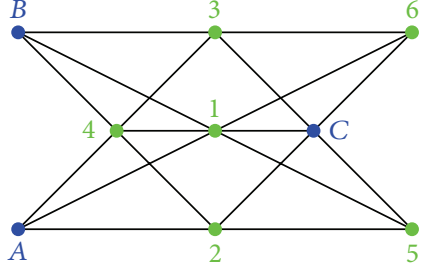

Figure 5: The Pappus theorem derived from the toric map.

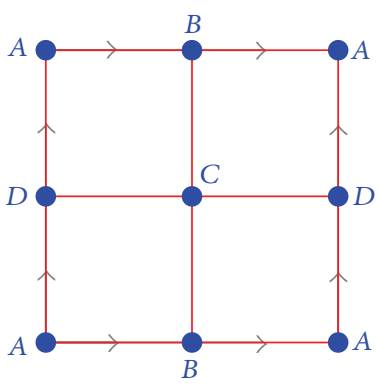

(a)

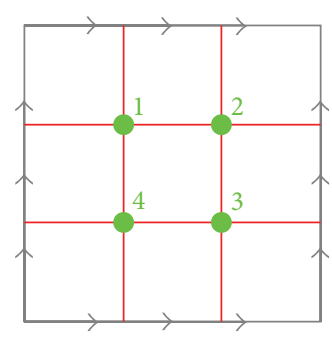

(b)
FIgURE 6: The toroidal Möbius graph $2 C_{4}$ and its dual $2 C_{4}$.

The eight blocks obtained from the edges of the graph are

$$
\begin{aligned}
& \overline{A B} 41=C D 41, \\
& \overline{A B} 23=C D 23, \\
& \overline{B C} 12=A D 12, \\
& \overline{B C} 34=A D 34, \\
& \overline{C D} 23=A B 23, \\
& \overline{C D} 41=A B 41, \\
& \overline{D A} 34=B C 34, \\
& \overline{D A} 12=B C 12 .
\end{aligned}
$$

There are many references for this configuration; see $[2,3,5,16-20]$. Perhaps the easiest way to construct this configuration in space is to first construct a $4 \times 4$ grid of eight lines; see Figure 7. The eight "Möbius" points can be eight points grouped in two lots of four as in the figure. The planes then correspond to the remaining eight points on the grid. A recent observation by the author [21] is that one can find three four by four matrices with the same 16 variables such that their determinants sum to zero, and it is closely related to the fact that there are certain three quadratic surfaces in space associated with this configuration. See [16] for a discussion of the three quadrics.

4.4. The Non-Möbius $8_{4}$ Configurational Theorem. The eight points of the "other" $8_{4}$ configurational theorem in $3 \mathrm{~d}$ space can be abstractly considered to be the members of the set

$$
\{0=A, 2=B, 4=C, 6=D, 1,3,5,7\},
$$




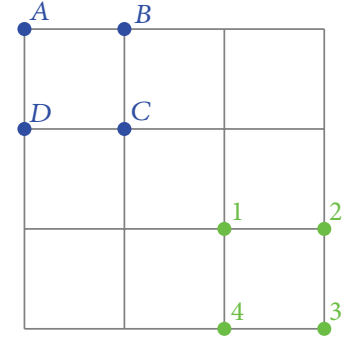

FIgURE 7: The Möbius $8_{4}$ configuration on eight lines.

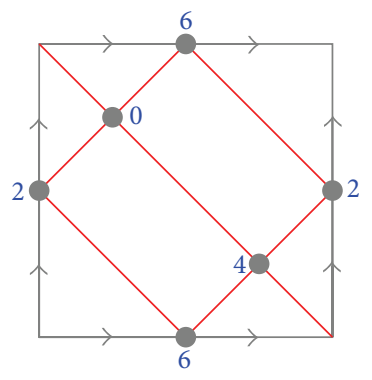

(a)

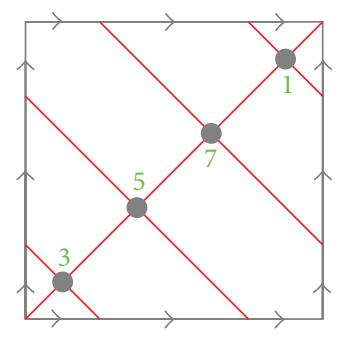

(b)
Figure 8: The toroidal graph $K_{4}+2 e$ and its dual $K_{4}+2 e$.

while the eight blocks (contained in planes when the configuration is embedded in $3 \mathrm{~d}$ space) are in correspondence with the eight edges of the $K_{4}+2 e$ graph which has four vertices: it can be constructed as the complete graph on four vertices plus two other nonadjacent edges.

The eight blocks obtained from the edges of the graph are

$$
\begin{aligned}
& \overline{C D} 14=0215=0125, \\
& \overline{A C} 13=2613=1236, \\
& \overline{A D} 37=2437=2347, \\
& \overline{B D} 35=0435=3450, \\
& \overline{A B} 15=4615=4561, \\
& \overline{A C} 57=2657=5672, \\
& \overline{B C} 37=0637=6703, \\
& \overline{B D} 17=0417=7014 .
\end{aligned}
$$

The standard cyclic representation of this configuration is that the points are the integers modulo eight, while the blocks are the subsets $\{0,1,2,5\}+i(\bmod 8)$; see Glynn [3] and Figure 8. As with the Möbius configuration, the configuration can always be constructed on a $4 \times 4$ grid of lines; see Figure 9. The planes then correspond to the remaining eight points on the grid.

4.5. The Gallucci Theorem. Consider Figures 10 and 11. The twelve points of the Gallucci configuration in $3 \mathrm{~d}$ space

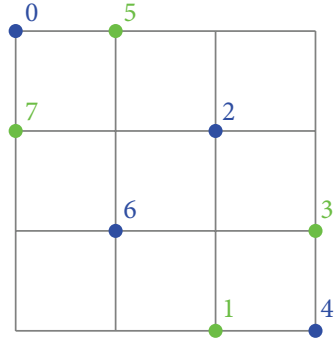

FIGURE 9: The other $8_{4}$ configuration on eight lines.

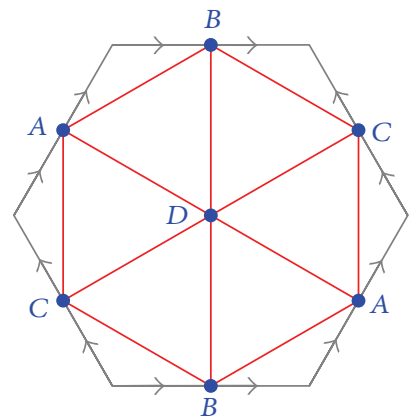

(a)

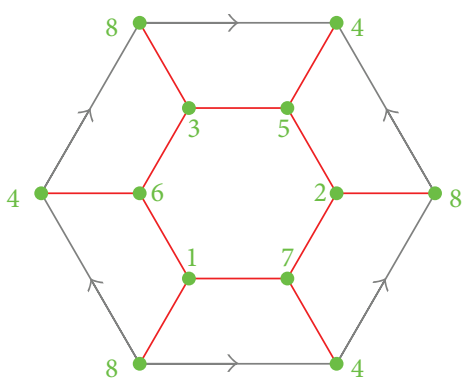

(b)

FIGURE 10: The toroidal Gallucci graph $2 K_{4}$ and its dual, cube graph.

are $A, B, C, D, 1, \ldots, 8$, while the twelve blocks (contained in planes when the configuration is in $3 \mathrm{~d}$ space) are in correspondence with the twelve points on the $4 \times 4$ grid other than $A, B, C, D$. Note that we are representing the torus as a hexagon with opposite sides identified. This is just an alternative to the more common representation of the torus as a rectangle with opposite sides identified. The arrows on the outside of the hexagons show the directions for which the identifications are applied. (The hexagons' boundaries are not graph edges.)

Another thing to note is that the only place the author has seen the name "Gallucci" attached to this configuration is in the works of Coxeter; see [2, Section 14.8]. The theorem appears in Baker's book [5, page 49], which appeared in its first edition in 1921, well before Gallucci's major work of 1928; see [18]. Due to its fairly basic nature it was obviously known to geometers of the 19th century. However, in deference to Coxeter, we are calling it "Gallucci's theorem." 


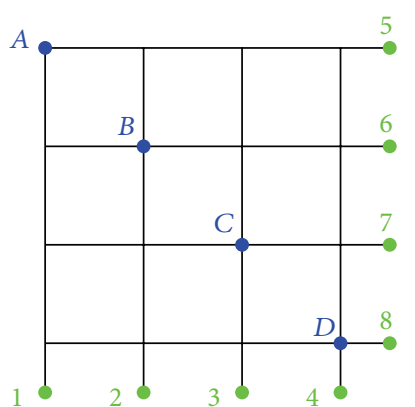

FIgURE 11: The Gallucci theorem of eight lines in 3d space.

The Gallucci configuration is normally thought of as a collection of eight lines, but here we are obtaining it from certain subsets of points and planes related to it. One set of four mutually skew lines is generated by the pairs of points $A 1, B 2, C 3, D 4$, and the other set of four lines by the four pairs $A 5, B 6, C 7, D 8$.

The twelve blocks obtained from the edges of the graph are

$$
\begin{array}{lll}
\overline{C D} 25=A B 25, & \overline{B D} 35=A C 35, & \overline{B C} 45=A D 45, \\
\overline{A D} 36=B C 36, & \overline{A C} 46=B D 46, & \overline{A B} 47=C D 47, \\
\overline{C D} 16=A B 16, & \overline{B D} 17=A C 17, & \overline{B C} 18=A D 18, \\
\overline{A D} 27=B C 27, & \overline{A C} 28=B D 28, & \overline{A B} 38=C D 38 .
\end{array}
$$

Some practical considerations remain: small graphs may determine relatively trivial properties of space, but we have seen in our examples that many graphs correspond to fundamental and nontrivial properties. We also obtain an automatic proof for these properties just from the embedding onto the surface. For some graphs on orientable surfaces the constructed geometrical configuration must collapse into smaller dimensions upon embedding into space or have points or hyperplanes that merge. This is a subject for further investigation.

\section{References}

[1] S. Lavietes, New York Times obituary, 2003, http://www.nytimes.com/2003/04/07/world/harold-coxeter-96-who-foundprofound-beauty-in-geometry.html.

[2] H. S. M. Coxeter, Introduction to Geometry, John Wiley \& Sons, New York, NY, USA, 1961.

[3] D. G. Glynn, "Theorems of points and planes in three-dimensional projective space," Journal of the Australian Mathematical Society, vol. 88, no. 1, pp. 75-92, 2010.

[4] P. Dembowski, Finite Geometries, vol. 44 of Ergebnisse der Mathematik und ihrer Grenzgebiete, Springer, New York, NY, USA, 1968.

[5] H. F. Baker, Principles of Geometry, vol. 1, Cambridge University Press, London, UK, 2nd edition, 1928.

[6] D. Hilbert, Grundlagen der Geometrie, Göttingen, 1899.
[7] D. G. Glynn, "A note on $N_{K}$ configurations and theorems in projective space," Bulletin of the Australian Mathematical Society, vol. 76, no. 1, pp. 15-31, 2007.

[8] S. Huggett and I. Moffatt, "Bipartite partial duals and circuits in medial graphs," Combinatorica, vol. 33, no. 2, pp. 231-252, 2013.

[9] L. Heffter, "Ueber das Problem der Nachbargebiete," Mathematische Annalen, vol. 38, no. 4, pp. 477-508, 1891.

[10] J. R. Edmonds, "A combinatorial representation for polyhedral surfaces," Notices of the American Mathematical Society, vol. 7, article A646, 1960.

[11] B. Bollobás and O. Riordan, "A polynomial invariant of graphs on orientable surfaces," Proceedings of the London Mathematical Society, vol. 83, no. 3, pp. 513-531, 2001.

[12] B. Bollobás and O. Riordan, "A polynomial of graphs on surfaces," Mathematische Annalen, vol. 323, no. 1, pp. 81-96, 2002.

[13] G. A. Jones and D. Singerman, "Theory of maps on orientable surfaces," Proceedings of the London Mathematical Society, vol. 37, no. 2, pp. 273-307, 1978.

[14] J. Dieudonné, "Les déterminants sur un corps non commutatif," Bulletin de la Société Mathématique de France, vol. 71, pp. 27-45, 1943.

[15] I. Gelfand, S. Gelfand, V. Retakh, and R. L. Wilson, "Quasideterminants," Advances in Mathematics, vol. 193, no. 1, pp. 56141, 2005.

[16] W. Blaschke, Projektive Geometrie, Birkhäuser, Basel, Switzerland, 3rd edition, 1954.

[17] H. S. M. Coxeter, "Self-dual configurations and regular graphs," Bulletin of the American Mathematical Society, vol. 56, pp. 413$455,1950$.

[18] G. Gallucci, Complementi di geometria proiettiva. Contributo alla geometria del tetraedro ed allo studio delle configurazioni, Università degli Studi di Napoli, Napoli, Italy, 1928.

[19] A. F. Moebius, "Kann von zwei dreiseitigen Pyramiden eine jede in Bezug auf die andere um- und eingeschrieben zugleich heissen?" Crelle's Journal für die reine und angewandte Mathematik, vol. 3, pp. 273-278, 1828.

[20] A. F. Moebius, "Kann von zwei dreiseitigen Pyramiden eine jede in Bezug auf die andere um- und eingeschrieben zugleich heissen?" Gesammelte Werke, vol. 1, pp. 439-446, 1886.

[21] D. G. Glynn, "A slant on the twisted determinants theorem," Submitted to Bulletin of the Institute of Combinatorics and Its Applications. 


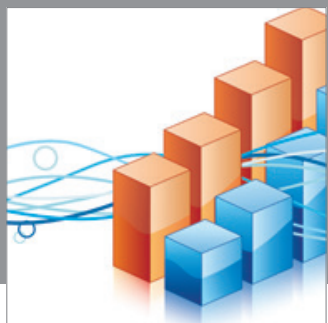

Advances in

Operations Research

mansans

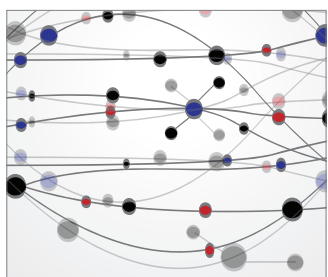

The Scientific World Journal
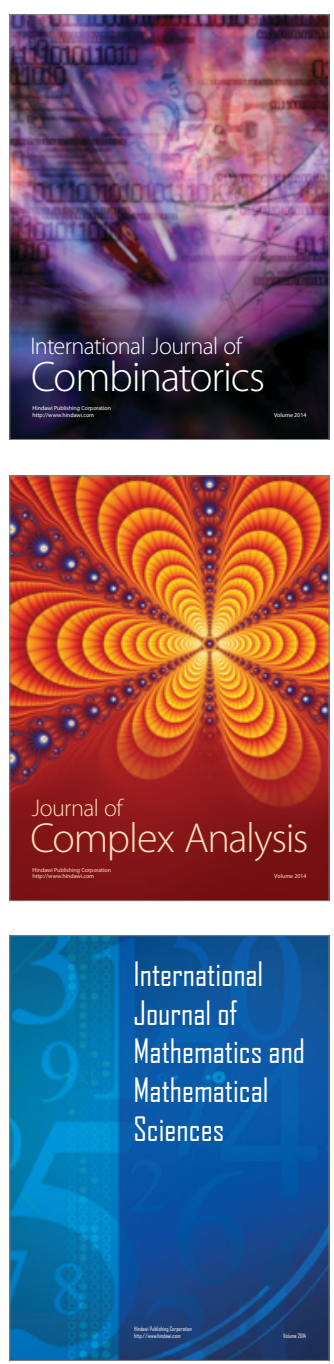
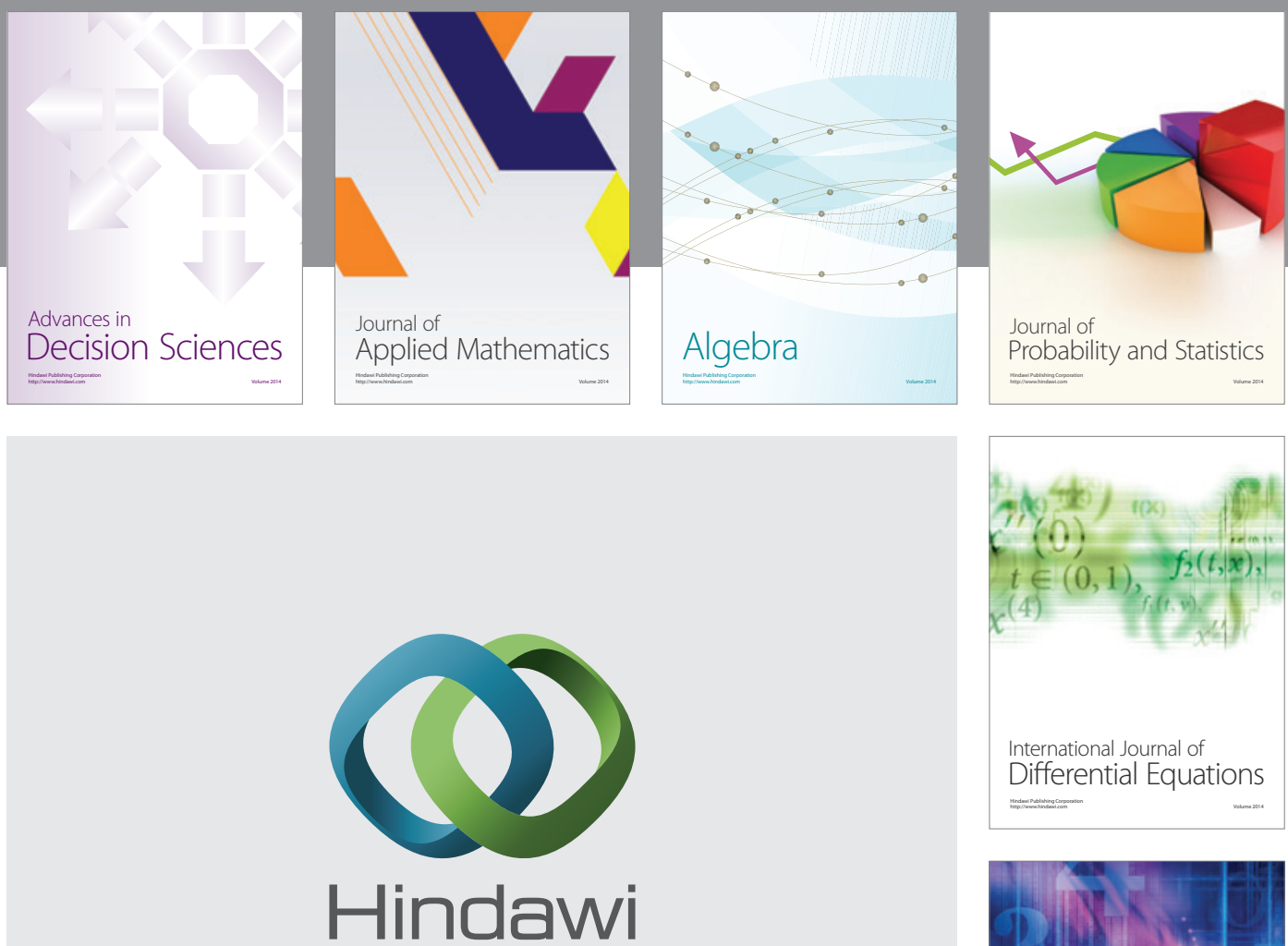

Submit your manuscripts at http://www.hindawi.com
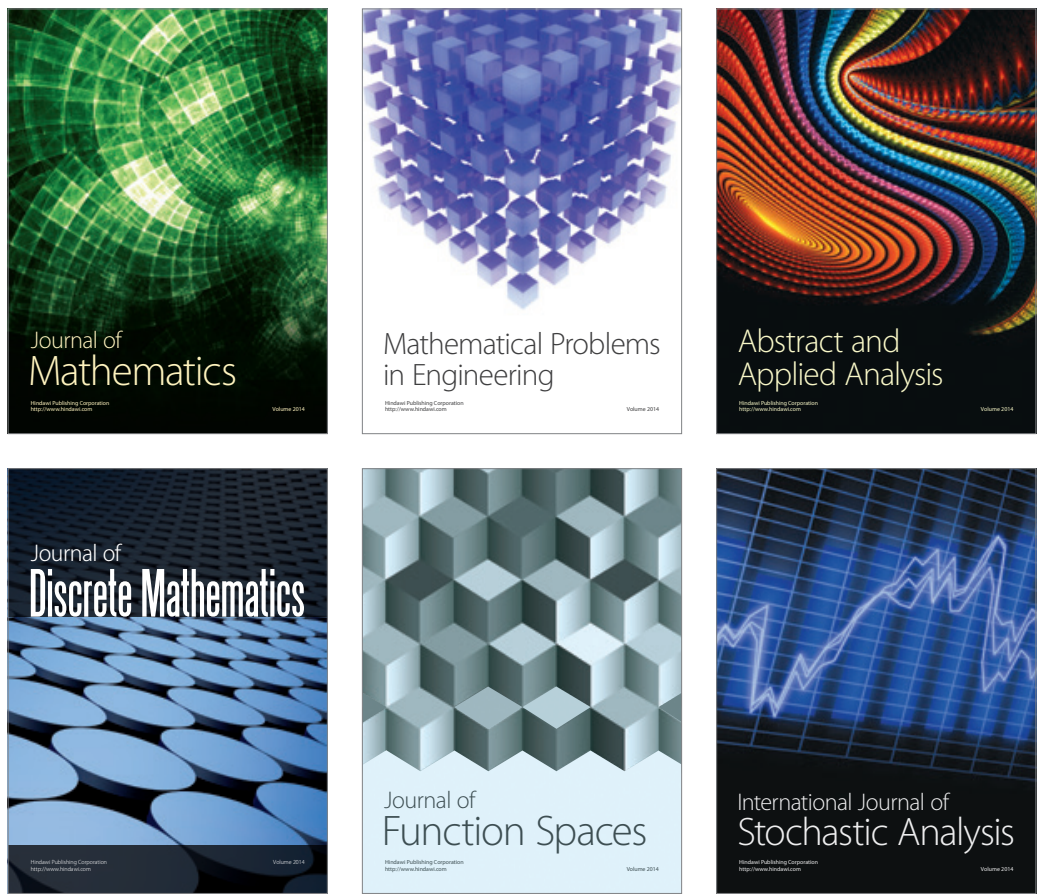

Journal of

Function Spaces

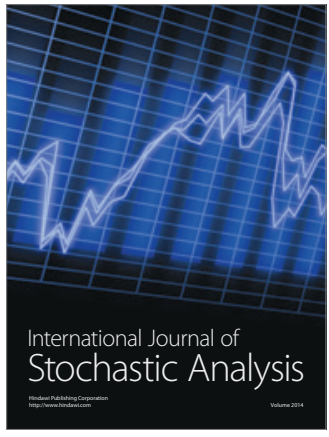

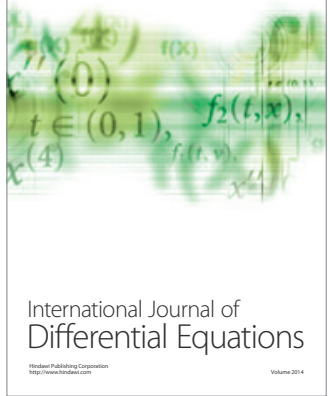
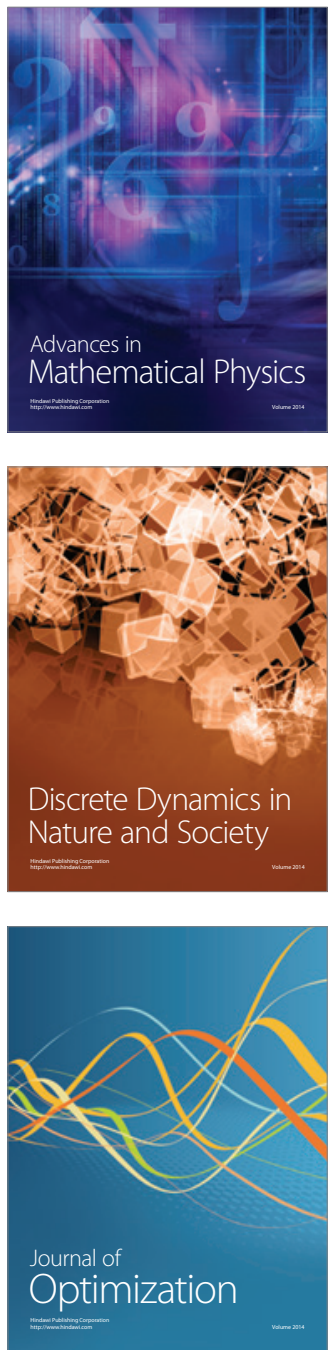